\title{
Preface to the Special Issue of Photocatalysis for Solar Fuels
}

As natural photosynthesis does, direct conversion of solar energy into the storable form of chemical energy is an intriguing technology for solar energy utilization. Due to increasing concerns of energy and environmental problems caused by the consumption of fossil fuels, production of chemical solar fuels (such as hydrogen, methanol or methane, etc) by artificial photosynthesis, namely photocatalytic splitting of water or reduction of $\mathrm{CO}_{2}$, has been a hot research topic in recent years.

In order to efficiently convert solar energy into chemical energy of solar fuels, development of efficient photocatalysts and better understanding the fundamental photocatalytic reaction processes for water splitting and $\mathrm{CO}_{2}$ reduction are in highly demanding. To do so, appropriate light absorption photocatalysts (such as semiconductors), oxygen evolution catalysts (OER), hydrogen evolution catalysts (HER) and $\mathrm{CO}_{2}$ reduction catalysts, as well as the mechanism of energy conversion processes have to be extensively studied. Some recent research progresses in these aspects, mainly from China, have been collected in this special issue of photocatalysis for solar fuels.

This special issue collects 23 papers, including three review papers. Liwu Zhang and coworkers reviewed enhancement of light absorption and utilization by photonic slow photons. Zhiliang Wang and coworkers reviewed recent progress in designing effective photoelectrodes for water splitting. Hongmei $\mathrm{Yu}$ and coworkers reviewed water electrolysis based on renewable energy for hydrogen production.

As for the HER catalysts and reactions, Tierui Zhang and coworkers contributed their work on $\mathrm{Sn}_{2} \mathrm{Nb}_{2} \mathrm{O}_{7}$ nanocrystals, Yujie Xiong and coworkers on $\mathrm{Au}-\mathrm{TiO}_{2}$ plasmonic photocatalysts, Xinheng Li and coworkers on PdS-CdSe@CdS-Au nanorods, Xiaoxiang $\mathrm{Xu}$ and coworkers on $\mathrm{Ti}^{3+}$ modified anatase $\mathrm{TiO}_{2}$, Meiyin Liu and coworkers on $\mathrm{Zn}_{0.9} \mathrm{Cd}_{0.1} \mathrm{~S}$ solid solution, Geng-geng Luo and coworkers on fac- and mer-tris(thiosemicarbazide) cobalt(III), Baojun $M a$ and coworkers on noble metal free phosphide/CdS composite photocatalyst, Yamei Li and coworkers on $\mathrm{MoS}_{2}$ HER at Neutral $\mathrm{pH}$, and Jingjun Ma and coworkers on surface defects doping of ultrafine $\mathrm{TiO}_{2}$ with transition metal to enhance the photocatalytic $\mathrm{H}_{2}$ production activity.

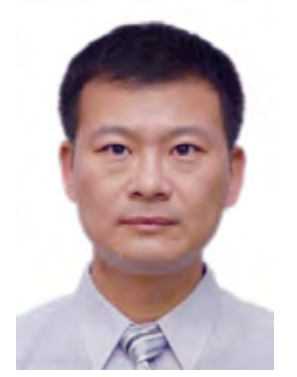

Prof. Hongxian Han (Guest Editor)

State Key Laboratory of Catalysis,

Dalian National Laboratory for Clean Energy,

Dalian Institute of Chemical Physics,

Chinese Academy of Sciences;

School of Future Technology,

University of Chinese Academy of Sciences

E-mail: hxhan@dicp.ac.cn

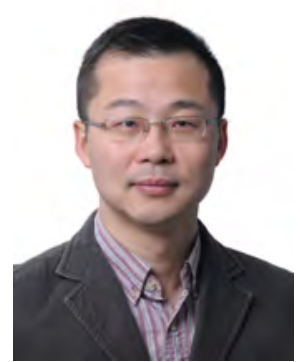

Prof. Gang Liu (Guest Editor)

Shenyang National Laboratory for Materials Science,

Institute of Metal Research,

Chinese Academy of Sciences,

College of Materials Science and Engineering, University of Science and Technology of China E-mail: gangliu@imr.ac.cn 
As for the OER catalysts and reactions, Yong Ding and coworkers contributed their work on dicobalt(III) polypyridine, Fei Li and coworkers on nickel oxides derived from molecular precursor and on binuclear Ru-bda catalyst, Shaohua Shen and coworkers on Co,Ni-metallated dicyanamide modified $\mathrm{BiVO}_{4}$, Yan Gao and coworkers on copper oxide film in situ-generated from $\mathrm{Cu}(\mathrm{II})$ tricine complex, Wansheng You and coworkers on $\left[\mathrm{Ag}\left(\mathrm{H}_{2} \mathrm{O}\right)\left(\mathrm{H}_{3} \mathrm{PW}_{11} \mathrm{O}_{39}\right)\right]^{3-}$ on $\mathrm{TiO}_{2}$ anode for water oxidation), and Gang Liu and coworkers on homogenous boron doped $\mathrm{TiO}_{2}$ shell supported on a $\mathrm{TiB}_{2}$ core for enhanced photocatalytic water oxidation.

As for the overall water splitting reactions, Xinchen Wang and coworkers contributed their work on Type-II heterojunction $\mathrm{TiO}_{2}$ photoanode for photoelectrochemical water splitting. Wenfeng Shangguan and coworkers on Zinc-doped g- $\mathrm{C}_{3} \mathrm{~N}_{4} / \mathrm{BiVO}_{4} \mathrm{Z}$-scheme Photocatalysis for overall water splitting, and Hongxian Han and coworkers on $\mathrm{Pt}-\mathrm{SrTiO}_{3}(\mathrm{La}, \mathrm{Cr}) / \mathrm{PtO}_{x}-\mathrm{WO}_{3} \mathrm{Z}$-scheme system for overall water splitting. Peng Kang and coworkers contributed their work on photoelectrocatalytic $\mathrm{CO}_{2}$ Reduction by Cobalt Complexes at
Silicon Electrode, and Lizhu Wu and coworkers contributed their work on photoinduced reductive cross coupling of aldehydes, ketones and Imines with arenes for aryl substituted alcohols and amines.

Though the papers presented in this special issue cannot stand for the top research works on photocatalysis for solar fuels, it can indeed be served as a peer for the on-going research activities on solar fuels research in China. We hope that this special issue may contribute to the development of more efficient photocatalysts and systems, spark more brilliant ideas, gain more attention on the research of artificial photosynthesis of solar fuels from the scientific community and the public, and attract more scholars to involve in the research of this "holy grail of sciences".

As the Guest Editors of this special issue, we would like to express our sincere thanks to all the authors who contributed to this special issue and all the reviewers for their timely evaluation of the submitted manuscripts. We are indebted to the editorial staff for their hardworking in editing and publishing this special issue. 